\title{
Sonic hedgehog secreted by neurons regulates angiopoietin expression in neighboring fibroblasts
}

\author{
WOO JEAN KIM ${ }^{1}$, SAE-WON LEE ${ }^{2,3}$ and KYU-WON KIM ${ }^{4,5}$ \\ ${ }^{1}$ National Research Laboratory of Regenerative Sexual Medicine, Department of Urology, \\ Inha University School of Medicine, Incheon; ${ }^{2}$ Department of Internal Medicine and Innovative \\ Research Institute for Cell Therapy; ${ }^{3}$ Biomedical Research Institute, Seoul National University Hospital; \\ ${ }^{4}$ Division of Pharmaceutical Biosciences, Research Institute of Pharmaceutical Sciences, College of Pharmacy, \\ ${ }^{5}$ WCU Department of Molecular Medicine and Biopharmaceutical Sciences, \\ Seoul National University, Seoul, Republic of Korea
}

Received January 22, 2014; Accepted April 25, 2014

DOI: $10.3892 / \mathrm{ijmm} .2014 .1767$

\begin{abstract}
Lately, the importance of the communication between different cell types and the understanding of the cell communication pathway has been emphasized, as it may provide a novel therapeutic strategy for the regeneration of damaged tissue. In the present study, we suggest that sonic hedgehog (Shh) is a mediator of cell communication between neurons and fibroblasts. Recombinant Shh (rShh) affected the expression of the angiogenic factors, angiopoietin (Ang)-1 and Ang-2, in fibroblasts, but not in neurons or neural progenitor cells (NPCs). The expression of the Shh downstream transcription factor, Gli1, was markedly increased in neurons and NPCs, indicating that neurons and NPCs responded to rShh. However, rShh did not affect Ang-1 and Ang-2 expression in neurons and NPCs. It should be noted that Shh was strongly expressed in neurons, but that Shh expression was undetectable in fibroblasts. We performed a co-culture assay using neurons and fibroblasts to investigate whether the expression of Ang-1 and Ang-2 is regulated by cell communication, without rShh treatment. Ang-1 expression in fibroblasts was markedly upregulated by co-culture with neurons, whereas Ang-2 expression was decreased by co-culture with neurons. Moreover, when an Shh-neutralizing antibody was added, this effect was diminished. Collectively, our data suggest that Shh-expressing neurons regulate angiopoietin expression in neighboring fibroblasts in a paracrine manner.
\end{abstract}

Correspondence to: Dr Sae-Won Lee, Biomedical Research Institute, Seoul National University Hospital, 101 DaeHak-ro, JongRo-gu, Seoul 110-744, Republic of Korea

E-mail: sawolee@snu.ac.kr

Key words: co-culture, neutralizing antibody, cell-cell cross talk, growth factors

\section{Introduction}

Sonic hedgehog (Shh) is a secretory glycoprotein and acts as an autocrine and paracrine factor (1). Shh binds to its receptor, patched 1 (Ptch1). In conditions in which Shh is inactivated, Ptch1 inhibits the action of the receptor, Smoothened. When Shh binds to Ptch1, this suppression of Smoothened is reversed, which in turn activates the transcription factor, Gli1 $(1,2)$. Gli1 upregulates several downstream signaling genes, including Ptch1 and Gli1 $(3,4)$. Shh plays an important role in the developmental process, which includes the regulation of axonal guidance, cell differentiation and the proliferation of neural progenitor cells (NPCs) (5-7). In adults, Shh, is involved in hypoxia-induced neural progenitor proliferation in the brain injured by stroke (8), controls stem cell maintenance in postnatal and adult brain neurogenesis (9), regulates angiogenesis and vasculogenesis $(10,11)$ and strengthens vascular tightness (12).

Angiopoietin (Ang)-1 and Ang-2 belong to the angiopoietin family, and play an important role in blood vessel formation both in normal development and pathological conditions $(13,14)$. The balance between Ang-1 and Ang-2 is important for the maintenance of the vasculature network (15). Ang-1 upregulates vascular integrity by modulating tight junction proteins $(16,17)$, enhances endothelial regeneration in diabetic mice (18) and recovers ischemic limb injury through the recruitment of bone marrow-derived progenitor cells (19). In addition to the role of Ang-1 and Ang-2 in angiogenesis, previous studies have shown that they have a broad target cell spectrum. Ang-1 blocks cell death and improves the survival of fibroblasts, skeletal myocytes and cardiomyocytes (20-22). It also induces neurite outgrowth (23), as well as the proliferation and differentiation of NPCs (24). Ang-2 also regulates NPC differentiation and migration (25). These reports indicate the importance of angiopoietin as a broad spectrum regulatory factor.

We have previously reported that Shh affects Ang-1 and Ang-2 mRNA expression only in fibroblasts (26). In the present study, we demonstrate that Shh is produced by neurons, and that the mRNA expression of Ang-1 and Ang- 2 in fibroblasts is 
modulated by co-culture with neurons, without exogenous Shh treatment. Moreover, Shh-neutralizing antibody significantly blocked the regulation of Ang-1 and Ang-2 in fibroblasts which was induced by co-culture with neurons. Our data suggest that fibroblasts and neurons communicate with each other through Shh signaling. Thus, we propose the concept of fibroblast/ neuron cross-talk.

\section{Materials and methods}

Animals, cell isolation and culture of neural progenitors and neurons. All the animal experiments were performed under the approval of the Institutional Animal Care and Use Subcommittee of Inha University Hospital (Incheon, Korea). For the culture of fresh NPCs, the cortices of fetuses obtained from CD1 mice [embryonic day (E)14-16] were isolated, minced and incubated in a PBS solution with $0.25 \%$ trypsin and $0.01 \%$ DNase I for $20 \mathrm{~min}$ as previously described (26). The cells were resuspended in DMEM/F12 medium (Gibco/ Invitrogen, Carlsbad, CA, USA) containing 1\% FBS, N2 supplement (Gibco/Invitrogen), 0.6\% glucose, HEPES (50 mmol/l), bFGF (20 ng/ml), EGF $(20 \mathrm{ng} / \mathrm{ml})$, heparin $(5 \mu \mathrm{g} / \mathrm{ml})$ and penicillin/streptomycin/amphotericin B (1:100 dilution; Gibco/ Invitrogen). The cells were plated at $2 \times 10^{5}$ cells/well into 24 -well plates coated with poly-D-lysine and laminin (PDL/L plates; Biocoat Inc., Horsham, PA, USA; BD Biosciences, Franklin Lakes, NJ, USA). After 24 h, 1\% FBS was removed. For the neuron cultures, mouse cortices (E15-17) were harvested as described in our previous study (26). The cells were seeded at $3 \times 10^{5}$ cells/well into PDL/L plates (Biocoat Inc.) and cultured in defined neuron culture medium; neurobasal medium (Gibco, Invitrogen) containing $2 \% \mathrm{~B} 27$, glutamine $(2 \mathrm{mmol} / \mathrm{l})$ and $1 \%$ penicillin/streptomycin. On days $1-3$, glutamate $(25 \mu \mathrm{g} / \mathrm{ml})$ and $\beta$-mercaptoethanol $(10 \mu \mathrm{mol} / \mathrm{l})$ were added. On day 3 , Ara-C (10 $\mu \mathrm{mol} / \mathrm{l})$ was added for $24 \mathrm{~h}$, and the medium was then exchanged with fresh defined neuron culture medium.

Cell line and reagents. NIH3T3 mouse embryonic fibroblasts were purchased [American Type Culture Collection (ATCC), Manassas, VA, USA] and cultured in DMEM containing 10\% FBS (Gibco/Invitrogen) and 1\% penicillin/streptomycin. Mouse recombinant Shh (mrShh) was purchased from GenScript (Piscataway, NJ, USA; Cat. no. Z03050) and the cells were incubated with 10 or $50 \mathrm{nM} \mathrm{rShh}$ for $16 \mathrm{~h}$. MAB4641, a neutralizing antibody against Shh $[10 \mu \mathrm{g} / \mathrm{m}$; R\&D Systems (Minneapolis, MN, USA)] was used for the specific blocking of Shh in the culture medium.

Quantitative (real-time) reverse transcription PCR (RT-qPCR). Total RNA was extracted using the QIAshredder and the RNeasy Mini kit (Qiagen, Hilden, Germany). Total RNA (1-2 $\mu \mathrm{g})$ was converted into cDNA using the PrimeScript ${ }^{\mathrm{TM}} 1$ st strand cDNA Synthesis kit (Takara Bio, Inc., Shiga, Japan). For real-time PCR assay, mouse Ang-1, Ang-2, Ptch1, Shh and Gli1 were analyzed with an ABI TaqMan Gene Expression Assay primer and FAM probe sets (Applied Biosystems, Foster City, CA, USA). Transcript levels were normalized to the $18 \mathrm{~S}$ rRNA. Real-time samples were run on an ABI PRISM-7500 sequence detection system (Applied Biosystems). In addition, end-point PCR for Ang-1 and Ang-2 was performed using following primers and GAPDH was used for normalization: Ang-1 forward, 5'-aaacagcaaatgggaacagg-3' and reverse, 5'-gggcaggtgaactccactaa-3' (melting temperature, $60^{\circ} \mathrm{C} ; 35$ cycles); Ang-2 forward, 5'-caaggcactgagagacacca- 3 ' and reverse, $5^{\prime}$-ctgaactcccacggaacatt- $3^{\prime}$ (melting temperature, $60^{\circ} \mathrm{C}$; 35 cycles); and GAPDH forward, 5'-ccactggcgtcttcaccac-3' and reverse, $5^{\prime}$-cctgcttcaccaccttcttg- $3^{\prime}$ (melting temperature, $60^{\circ} \mathrm{C}$; 27 cycles).

Western blot analysis. The cells were harvested and lysed in lysis buffer (40 mM Tris pH 7.4, $10 \mathrm{mM}$ EDTA, $120 \mathrm{mM}$ $\mathrm{NaCl}, 0.1 \% \mathrm{NP}-40$ ) containing protease inhibitors (Roche Diagnostics GmbH, Mannheim, Germany). Total protein (10-30 $\mu \mathrm{g})$ was fractionated in SDS-PAGE and immunoblotted with specific antibodies against Ang-1 (Novus Biologicals, Littleton, CO, USA) and Ang-2 (Novus Biologicals). Actin (Calbiochem, La Jolla, CA, USA) was used as an internal control. We used the Kodak Image Station 400R to detect the bands and Kodak Molecular Imaging Software version 4.0. The quantification of band intensity was analysed using TINA 2.0 (Raytest, Straubenhardt, Germany) and normalized to the intensity of actin.

Co-culture assay. Neurons and NIH3T3 fibroblasts were co-cultured as previously described with certain modifications (16). Fibroblasts were seeded at the bottom of a 12-well plate. Neurons were plated into a Transwell chamber at $2 \times 10^{5}$ cells/well (pore size, $0.4 \mu \mathrm{m}$; Corning Life Science, Tewksbury, MA, USA). The Transwell chamber with the confluent neurons was then placed in the 12-well plate and was further incubated for $16 \mathrm{~h}$ in the defined neuron culture medium. During co-culture, a neutralizing antibody against Shh (MAB4641; $10 \mu \mathrm{g} / \mathrm{ml}$; R\&D Systems) was added to the bottom well. Total RNA was extracted from the fibroblasts to evaluate the effects of Shh secreted from the neurons.

Statistical analysis. All the results are expressed as the means \pm standard deviation. The differences between the groups were compared by an unpaired t-test or one-way analysis of variance (ANOVA). P-values $\leq 0.05$ were considered to indicate statistically significant differences. All statistical analyses were performed using SPSS 17.0 software (SPSS Inc., Chicago, USA).

\section{Results}

$r$ Shh regulates the expression of Ang-1 and Ang-2 in fibroblasts, but not in neurons and NPCs. We determined the expression of Ang-1 and Ang-2 following treatment with recombinant sonic hedgehog (rShh) (Fig. 1A). Ang-1 mRNA expression was increased in fibroblasts only, whereas Ang-2 mRNA expression was decreased by rShh treatment. Ang-1 and Ang-2 mRNA expression showed no change in either the neurons or the NPCs. Glil mRNA levels significantly increased following treatment with $\mathrm{rShh}$ in all cell types, indicating that neurons and NPCs responded to rShh normally; however, rShh did not affect Ang-1 and Ang-2 mRNA expression in neurons and NPCs (Fig. 1A), which is in accordance with the results of our previous study (26). To confirm the effects of rShh on angiopoietin at the protein level we performed western blot anlaysis (Fig. 1B). Ang-1 protein expression showed a marked 
A
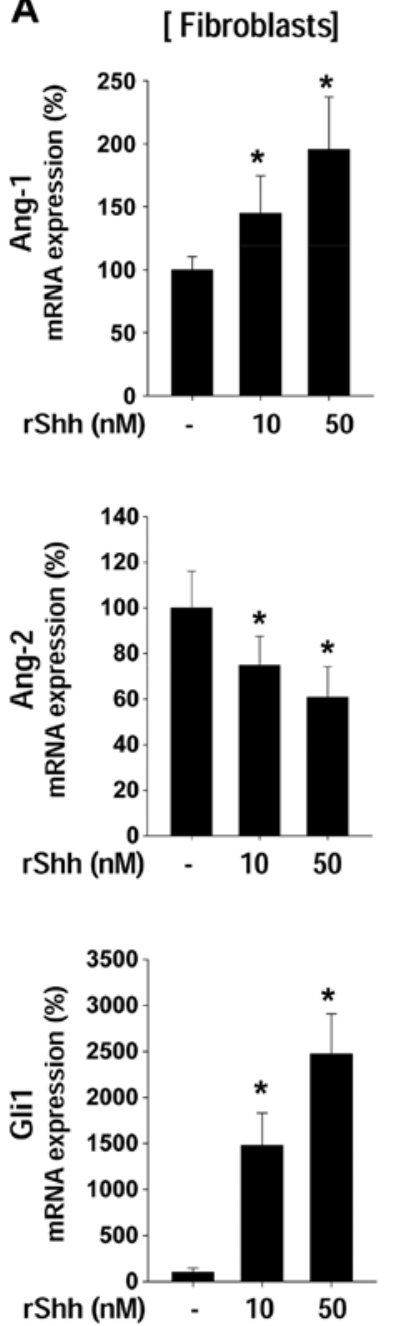

[ Neurons]
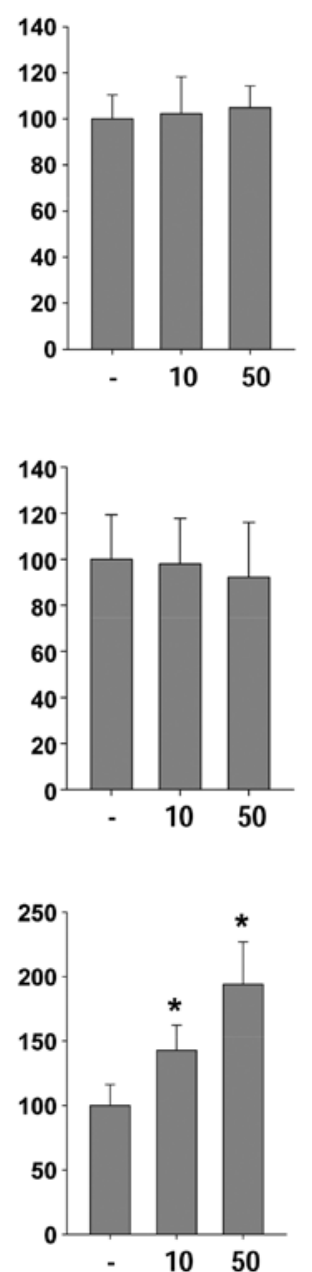

[NPCs]
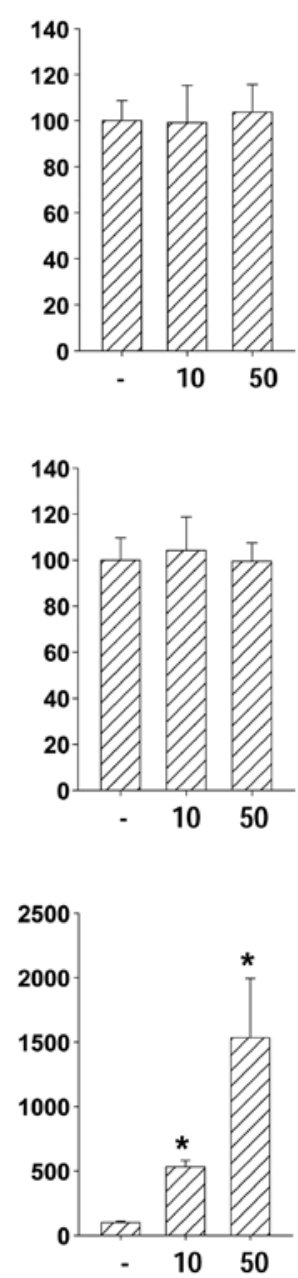

B
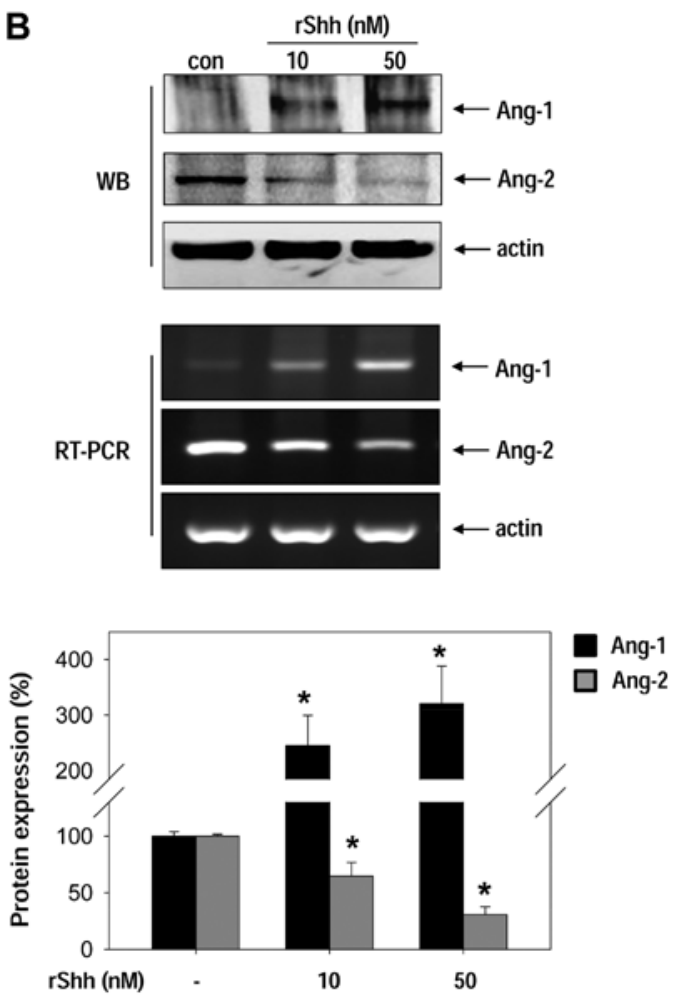

Figure 1. The expression of angiopoietin (Ang)-1 and Ang-2 is regulated by recombinant Shh (rShh) only in fibroblasts. (A) Fibroblasts, neurons and neural progenitor cells (NPCs) were incubated with 10 or $50 \mathrm{nM}$ rShh for $16 \mathrm{~h}$, and RT-qPCR was performed. Ang-1 mRNA expression was increased, whereas Ang-2 mRNA expression was decreased following treatment with rShh only in fibroblasts $(\mathrm{n}=5)$. Ang-1 and Ang-2 mRNA expression showed no change in either the neurons $(n=4)$ or the NPCs $(n=3)$, even though Gli1 mRNA expression was upregulated following treatment with $r S h h\left({ }^{*} p<0.001\right)$. (B) The expression of Ang-1 and Ang-2 in fibroblasts was confirmed by western blot analysis (WB; top panel, $\mathrm{n}=3$ ) and RT-qPCR (middle panel, $\mathrm{n}=3$ ). Quantitative graphs for western blot analysis (bottom panel, $\left.\mathrm{n}=3 ;{ }^{*} \mathrm{p}<0.05\right)$. Con, control (untreated cells).

increase in a dose-dependent manner, while Ang-2 expression decreased in a dose-dependent manner.

Shh-expressing cells and Shh-responsive cells differ. We then investigated Shh mRNA expression in 3 types of cells, fibroblasts, neurons and NPCs, in order to determine Shh basal levels (Fig. 2A). Shh was not expressed in fibroblasts, but it was expressed in both neurons and NPCs. Of note, Shh expression levels in the neurons were much higher than those in the NPCs (Fig. 2A). It is known that Shh acts in an autocrine and paracrine manner (1). Shh binds to its receptor, Ptch1, and activates its signaling with the upregulation of Ptch1 and Gli1 expression (3). Thus, we examined the mRNA expression of Ptch1 and Gli1 to clarify which cells are influenced by rShh (Fig. 2B and C). We hypothesized that rShh mainly affects neurons even though all 3 types of cells were affected by rShh, as endogenous Shh was highly synthesized in neurons. It should be noted however, that Shh downstream signaling, Ptch1 and Gli1, was upregulated in fibroblasts, but not in neurons (Fig. 2B and C). These data indicate that although neurons are one of the major cell sources for Shh expression, fibroblasts are the most responsive cells to Shh. Therefore, we suggest that Shh-expressing cells and Shh-responsive cells differ, and we suggest the existence of a cell cross-talk in Shh-mediated angiopoietin regulation.

Shh secreted by neurons regulates Ang-1 and Ang-2 gene expression in fibroblasts in a co-culture system. As shown in Fig. 2, we found that Shh expression was markedly increased in neurons, whereas Shh mainly affects fibroblasts as opposed to neurons. We suggested the existence of a cross-talk between neurons and fibroblasts. To confirm our hypothesis, we performed co-culture assay (Fig. 3). Fibroblasts were seeded at the bottom well of 12-well plate, and then a Transwell chamber with confluent neurons was placed in the 12-well plate (Fig. 3A). Following further incubation, total RNA was extracted from the fibroblasts and the Ang-1 and Ang-2 mRNA expression was determined (Fig. 3B-D). It is of interest to note that Ang-1 
A

\section{Shh mRNA}
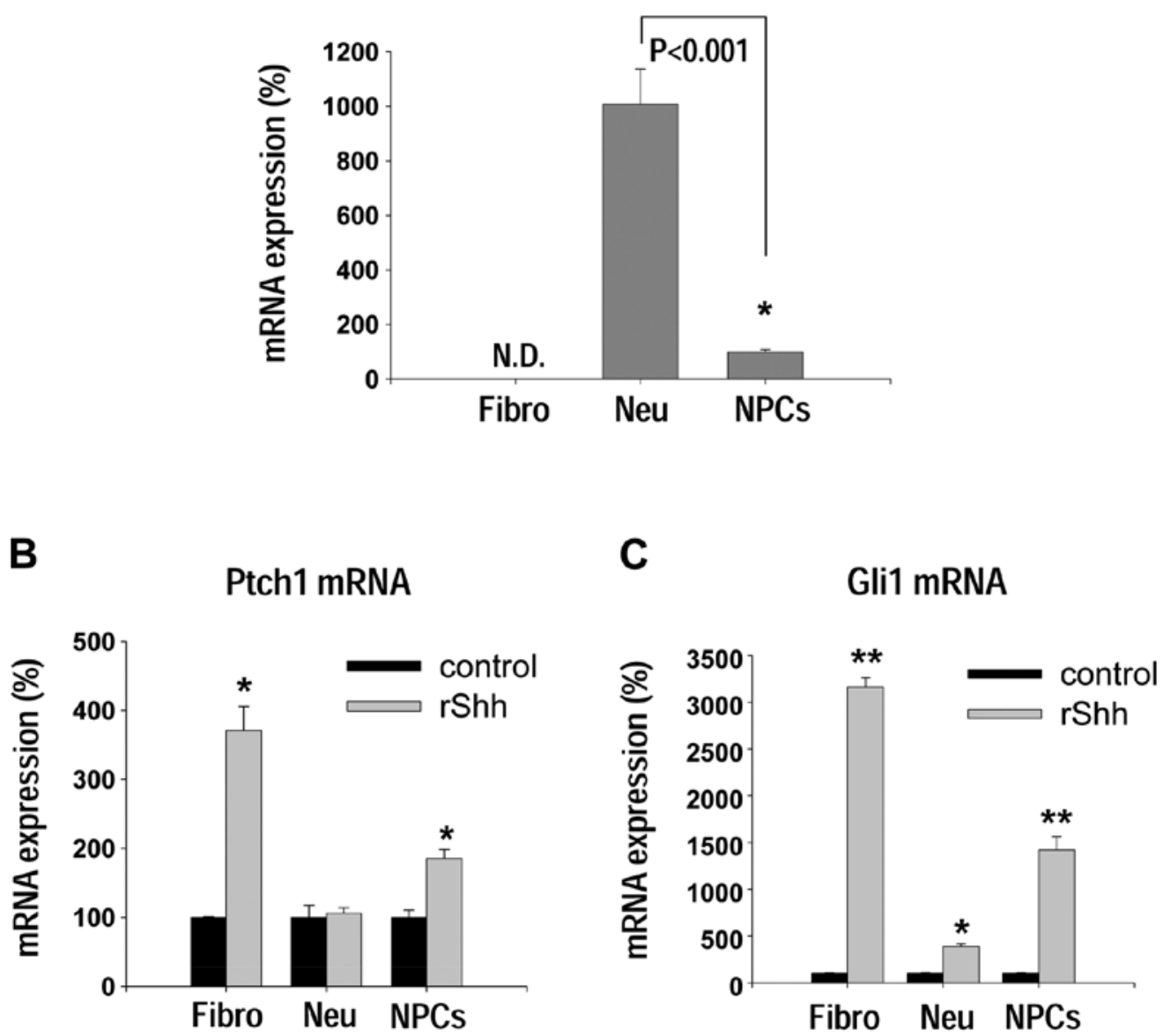

Figure 2. Sonic hedgehog (Shh) expression and the regulation of the Shh downstream targets, patched 1 (Ptch1) and Gli1, in 3 types of cells. (A) RT-qPCR for the determination of Shh mRNA expression revealed a high expression in neurons (Neu) (n=7). (B and C) Fibroblasts (Fibro), neurons and neural progenitor cells (NPCs) were treated with $50 \mathrm{nM}$ recombinant Shh (rShh) for $16 \mathrm{~h}$. The expresson of Ptch1 and Gli1, the downstream targets of Shh, was markedly increased following treatment with $\mathrm{rShh}$ in fibroblasts $\left(\mathrm{n}=3\right.$ each) $\left({ }^{*} \mathrm{p}<0.05,{ }^{* *} \mathrm{p}<0.005\right)$.

expression was markedly increased only when the neuron co-culture system was used (Fig. 3B). Moreover, the co-cultureinduced Ang-1 upregulation was markedly inhibited by the addition of a Shh-neutralizing antibody (MAB4641; Fig. 3B). On the other hand, Ang-2 mRNA expression in the fibroblasts decreased in the co-culture group; however, this reduction in Ang-2 expression following co-culture was reversed by the addition of Shh-neutralizing antibody (Fig. 3C). Our results suggest that Shh released from neurons, which may influence neighboring fibroblasts, thus regulating Ang-1 and Ang-2 expression (Fig. 3E).

\section{Discussion}

In this study, we found that neurons and fibroblasts communicate with each other and that the main source of Shh expression is from neurons, not fibroblasts or NPCs. In addition, the regulation of angiopoietin expression in fibroblasts was affected by neurons in the co-culture system, suggesting the existence of a cross-talk between neurons and fibroblasts. Lately, the importance of gaining an understanding of the communication pathway between different cell types has been emphasized in the research field of drug development. In the research area of neurovascular and oligovascular units, there is increasing evidence that there is a communication between blood vessels and brain cells $(27,28)$. Cerebral endothelial cells may secrete trophic factors that affect neighboring cells $(27,28)$. Likewise, astrocytes/endothelial coupling regulate the development of the cerebral vasculature, i.e., the blood-brain barrier, through Ang-1 (16). Nerves serve as a template for arteries and determine the arterial differentiation through the vascular endothelial growth factor $(29,30)$. Osteoblast-secreted Ang-1 promotes the survival and maintenance of hematopoietic stem cells (31). Endothelial cells stimulate fibroblast differentiation following ischemic injury and therefore increase tissue fibrosis following myocardial infarction (32). These studies have shown that the complex interplay between types of cells is extensively regulated in normal physiology.

As this cell cross-talk between cells may involve common growth factors and may be regulated in an interconnected manner, molecules, such as Shh hold great therapeutic potential. Shh upregulates vascularization and osteoblastic differentiation (33), and can also regulate both angiogenesis and myogenesis (34). Ang-1 decreases both vascular damage and cardiomyocyte death, thus improving cardiac function following myocardial infarction (17). Ang-1 also stimulates 
A

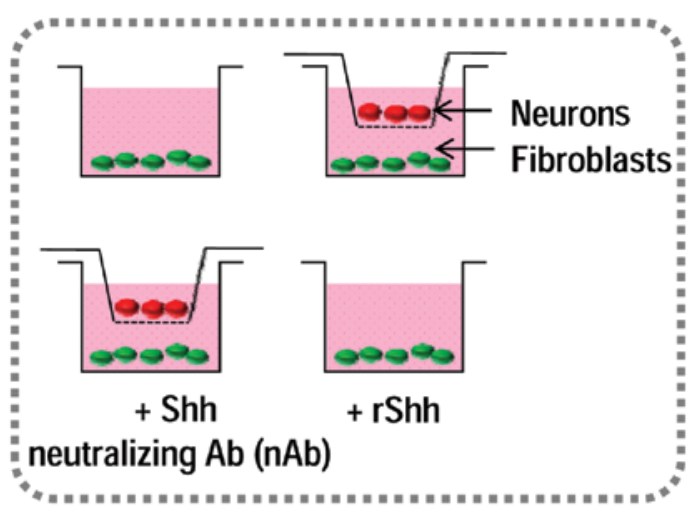

C

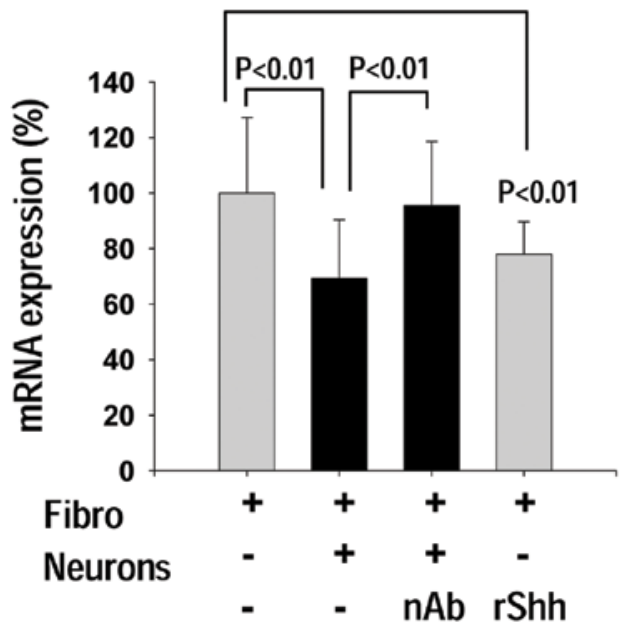

B

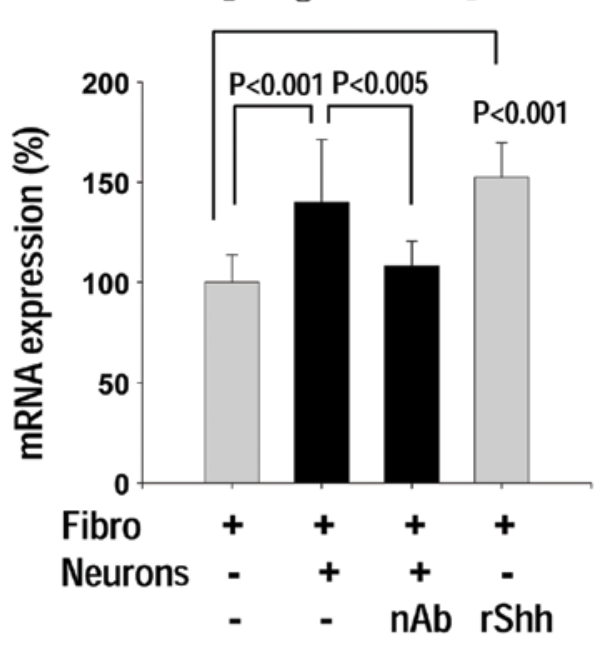

D

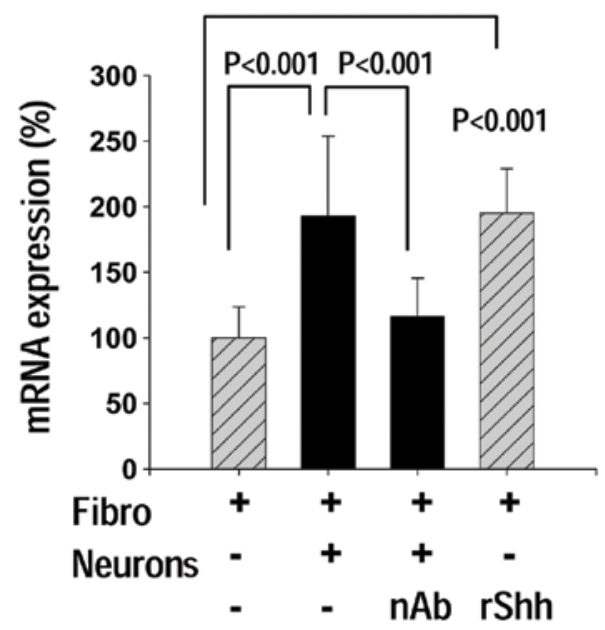

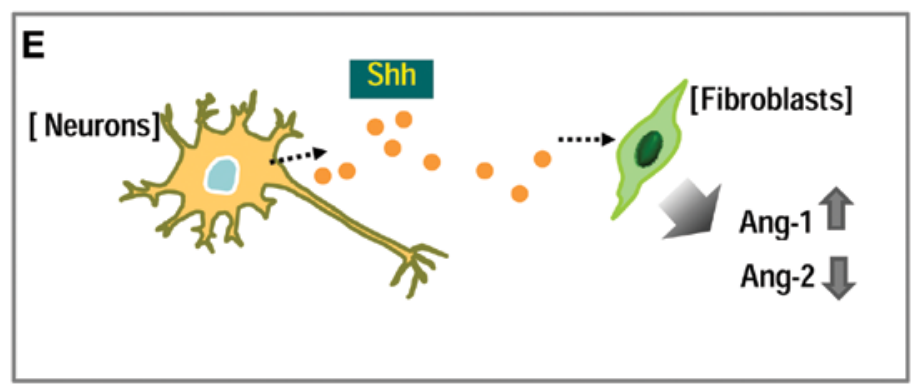

Figure 3. Neurons regulate angiopoietin (Ang)-1 and Ang-2 gene expression in fibroblasts through sonic hedgehog (Shh) secretion in a co-culture system. (A) Schematic illustration of co-culture between fibroblasts and neurons. Fibroblasts were seeded at the bottom of a 12-well plate. A Transwell chamber with confluent neurons was then placed in the 12-well plate and was further incubated. Total RNA was extracted from the fibroblasts and performed RT-qPCR was performed. (B and C) Ang-1 mRNA expression was increased by co-culture with neurons, whereas Ang-2 mRNA expression was decreased ( $\mathrm{n}=4$ ). Neutralizing antibody (nAb) against Shh (MAB4641, $10 \mu \mathrm{g} / \mathrm{ml}$ ) was added to the bottom well medium during co-culture, which blocked the effect of neurons on the expression of Ang-1 and Ang-2 mRNA in fibroblasts. Treatment of fibroblasts with recombinant Shh ( $\mathrm{rShh}$ ) protein (50 nM) was performed as a positive control. (D) The ratio of Ang-1 to Ang-2 expression was calculated (n=4). (E) Schematic diagram of cell cross-talk between fibroblasts and neurons in angiopoietin gene regulation. Shh was markedly in neurons. Shh was expressed and released from neurons, thus influencing neighboring fibroblasts to inversely regulate Ang-1 and Ang-2 expression through paracrine mechanisms. Fibro, fibroblasts.

muscle regeneration after muscle injury in mice by promoting muscle satellite cell self-renewal (35).
Moreover, previous studies have suggested that the combined transplantation of different cell types improves 
therapeutic potential. Vascular regeneration in hindlimb ischemia was shown to be enhanced by the transplantation of a combination of embryonic stem cell-derived endothelial and mural cells, compared with single-cell transplantation (36). The combination of Shh gene transfer and bone marrow-derived progenitor cells has also been shown to improve angiogenesis and muscle regeneration in limb ischemia to a greater degree than with each treatment individually (37). Therefore, an improved understanding of cell communication and the functions of regulatory cytokines, such as Shh may provide a novel strategy for the regeneration of damaged tissue.

\section{Acknowledgements}

This study was supported by the Basic Science Research Program through the National Research Foundation of Korea (NRF) funded by the Ministry of Science, ICT and Future Planning (2013R1A1A3012024, awarded to Sae-Won Lee), and by the Basic Science Research Program through the NRF grant funded by the Ministry of Education (NRF-2009-0076309, NRF-2011-0025506, awarded to Woo Jean Kim).

\section{References}

1. Rowitch DH, B SJ S-Jacques B, Lee SM, Flax JD, Snyder EY and McMahon AP: Sonic hedgehog regulates proliferation and inhibits differentiation of CNS precursor cells. J Neurosci 19: 8954-8965, 1999.

2. Taipale J, Cooper MK, Maiti T and Beachy PA: Patched acts catalytically to suppress the activity of Smoothened. Nature 418: 892-897, 2002.

3. Zedan W, Robinson PA, Markham AF and High AS: Expression of the Sonic Hedgehog receptor "PATCHED" in basal cell carcinomas and odontogenic keratocysts. J Pathol 194: 473-477, 2001.

4. Osterlund $\mathrm{T}$ and Kogerman P: Hedgehog signalling: how to get from Smo to Ci and Gli. Trends Cell Biol 16: 176-180, 2006.

5. Zhu G, Mehler MF, Zhao J, Yu Yung S and Kessler JA: Sonic hedgehog and BMP2 exert opposing actions on proliferation and differentiation of embryonic neural progenitor cells. Dev Biol 215: 118-129, 1999.

6. Wang YP, Dakubo G, Howley P, et al: Development of normal retinal organization depends on Sonic hedgehog signaling from ganglion cells. Nat Neurosci 5: 831-832, 2002.

7. Charron F, Stein E, Jeong J, McMahon AP and Tessier-Lavigne M The morphogen sonic hedgehog is an axonal chemoattractant that collaborates with netrin-1 in midline axon guidance. Cell 113 11-23, 2003.

8. Sims JR, Lee SW, Topalkara K, et al: Sonic hedgehog regulates ischemia/hypoxia-induced neural progenitor proliferation. Stroke 40: 3618-3626, 2009.

9. Palma V, Lim DA, Dahmane N, et al: Sonic hedgehog controls stem cell behavior in the postnatal and adult brain. Development 132: 335-344, 2005.

10. Pola R, Ling LE, Silver M, et al: The morphogen Sonic hedgehog is an indirect angiogenic agent upregulating two families of angiogenic growth factors. Nat Med 7: 706-711, 2001.

11. Surace EM, Balaggan KS, Tessitore A, et al: Inhibition of ocular neovascularization by hedgehog blockade. Mol Ther 13: 573-579, 2006.

12. Xia YP, He QW, Li YN, et al: Recombinant human sonic hedgehog protein regulates the expression of $\mathrm{ZO}-1$ and occludin by activating angiopoietin-1 in stroke damage. PLoS One 8: e68891, 2013.

13. Davis S, Aldrich TH, Jones PF, et al: Isolation of angiopoietin-1, a ligand for the TIE2 receptor, by secretion-trap expression cloning. Cell 87: 1161-1169, 1996.

14. Yancopoulos GD, Davis S, Gale NW, Rudge JS, Wiegand SJ and Holash J: Vascular-specific growth factors and blood vessel formation. Nature 407: 242-248, 2000.

15. Carmeliet P: Angiogenesis in health and disease. Nat Med 9: $653-660,2003$
16. Lee SW, Kim WJ, Choi YK, et al: SSeCKS regulates angiogenesis and tight junction formation in blood-brain barrier. Nat Med 9: 900-906, 2003.

17. Lee SW, Kim WJ, Jun HO, Choi YK and Kim KW: Angiopoietin-1 reduces vascular endothelial growth factor-induced brain endothelial permeability via upregulation of ZO-2. Int J Mol Med 23: 279-284, 2009.

18. Jin HR, Kim WJ, Song JS, et al: Intracavernous delivery of a designed angiopoietin-1 variant rescues erectile function by enhancing endothelial regeneration in the streptozotocin-induced diabetic mouse. Diabetes 60: 969-980, 2011.

19. Youn SW, Lee SW, Lee J, et al: COMP-Ang1 stimulates HIF-1alpha-mediated SDF-1 overexpression and recovers ischemic injury through BM-derived progenitor cell recruitment. Blood 117: 4376-4386, 2011.

20. Carlson TR, Feng Y, Maisonpierre PC, Mrksich M and Morla AO: Direct cell adhesion to the angiopoietins mediated by integrins. J Biol Chem 276: 26516-26525, 2001.

21. Dallabrida SM, Ismail N, Oberle JR, Himes BE and Rupnick MA: Angiopoietin-1 promotes cardiac and skeletal myocyte survival through integrins. Circ Res 96: e8-e24, 2005.

22. Lee SW, Won JY, Lee HY, et al: Angiopoietin-1 protects heart against ischemia/reperfusion injury through VE-cadherin dephosphorylation and myocardiac integrin-beta1/ERK/ caspase-9 phosphorylation cascade. Mol Med 17: 1095-1106, 2011.

23. Chen X, Fu W, Tung CE and Ward NL: Angiopoietin-1 induces neurite outgrowth of PC12 cells in a Tie2-independent, beta1-integrin-dependent manner. Neurosci Res 64: 348-354, 2009.

24. Rosa AI, Goncalves J, Cortes L, Bernardino L, Malva JO and Agasse F: The angiogenic factor angiopoietin-1 is a proneurogenic peptide on subventricular zone stem/progenitor cells. J Neurosci 30: 4573-4584, 2010.

25. Liu XS, Chopp M, Zhang RL, et al: Angiopoietin 2 mediates the differentiation and migration of neural progenitor cells in the subventricular zone after stroke. J Biol Chem 284: 22680-22689, 2009.

26. Lee SW, Moskowitz MA and Sims JR: Sonic hedgehog inversely regulates the expression of angiopoietin- 1 and angiopoietin-2 in fibroblasts. Int J Mol Med 19: 445-451, 2007.

27. Lok J, Gupta P, Guo S, et al: Cell-cell signaling in the neurovascular unit. Neurochem Res 32: 2032-2045, 2007.

28. Arai $\mathrm{K}$ and Lo EH: Oligovascular signaling in white matter stroke. Biol Pharm Bull 32: 1639-1644, 2009.

29. Mukouyama YS, Shin D, Britsch S, Taniguchi M and Anderson DJ: Sensory nerves determine the pattern of arterial differentiation and blood vessel branching in the skin. Cell 109: 693-705, 2002

30. Carmeliet $P$ and Tessier-Lavigne M: Common mechanisms of nerve and blood vessel wiring. Nature 436: 193-200, 2005.

31. Arai F, Hirao A, Ohmura M, et al: Tie2/angiopoietin-1 signaling regulates hematopoietic stem cell quiescence in the bone marrow niche. Cell 118: 149-161, 2004.

32. Lee SW, Won JY, Kim WJ, et al: Snail as a potential target molecule in cardiac fibrosis: paracrine action of endothelial cells on fibroblasts through snail and CTGF axis. Mol Ther 21: 1767-1777, 2013.

33. Dohle E, Fuchs S, Kolbe M, Hofmann A, Schmidt H and Kirkpatrick CJ: Sonic hedgehog promotes angiogenesis and osteogenesis in a coculture system consisting of primary osteoblasts and outgrowth endothelial cells. Tissue Eng Part A 16: 1235-1237, 2010

34. Straface G, Aprahamian T, Flex A, et al: Sonic hedgehog regulates angiogenesis and myogenesis during post-natal skeletal muscle regeneration. J Cell Mol Med 13: 2424-2435, 2009.

35. Abou-Khalil R, Le Grand F, Pallafacchina G, et al: Autocrine and paracrine angiopoietin 1/Tie-2 signaling promotes muscle satellite cell self-renewal. Cell Stem Cell 5: 298-309, 2009.

36. Yamahara K, Sone M, Itoh $\mathrm{H}$, et al: Augmentation of neovascularization [corrected] in hindlimb ischemia by combined transplantation of human embryonic stem cells-derived endothelial and mural cells. PLoS One 3: e1666, 2008.

37. Palladino M, Gatto I, Neri V, et al: Combined therapy with sonic hedgehog gene transfer and bone marrow-derived endothelial progenitor cells enhances angiogenesis and myogenesis in the ischemic skeletal muscle. J Vasc Res 49: 425-431, 2012. 